\title{
Laser-assisted microsurgery for intracordal cysts decreases cost-effectiveness
}

\author{
Frederik G. Dikkers
}

Received: 30 December 2010 / Accepted: 3 February 2011 / Published online: 18 February 2011

(c) The Author(s) 2011. This article is published with open access at Springerlink.com

Sir,

Matar et al. [1] nicely presented their results after $\mathrm{CO}_{2}$ laser-assisted microsurgery for intracordal cysts. The use of the laser in their clinic is widely acknowledged, recognized and respected. However, caution should be taken as one might think that laser-assisted microsurgery is the single best technique in treating intracordal cysts. As no comparison is made with the standard cold steel microsurgery, this is a question that remains unanswered.

We have published VHI results of patients with benign voice disorders [2], including cold steel microsurgery of intracordal cysts. Interestingly enough, the subjective functional results are very comparable. In our series of 68 patients, 8 were treated for intracordal cysts, 2 males and 6 females. Their VHI changed from $44 / 120$ preoperatively to 25/120 3 months postoperatively, where Matar et al. describe 51/120 and 28/120, respectively.

Some comments should be made. Results are presented quite roughly: the change in median $\mathrm{G}$ in the GRBAS has been presented without mentioning the mean $\mathrm{G}$. Improvement and trends were mentioned in changes of $\mathrm{R}, \mathrm{S}$, and $\mathrm{B}$ scale without offering exact data. Change in mean frequency range was presented in $\mathrm{Hz}$, while dealing with a population of a combination of males and females. As the $\mathrm{Hz}$ scale is a logarithmic one, it would have been better to present the change in frequency range in semitones, thereby abolishing gender differences.
The authors should be congratulated with the nice description of the surgical technique. However, laserassisted microsurgery is more expensive [3]. Laser-assisted microsurgery for intracordal cysts offers no clinical advantages. Comparable functional results can be obtained using cold steel instruments. In conclusion, laser-assisted microsurgery for intracordal cysts decreases cost-effectiveness.

\section{Conflict of interest There is no conflict of interest to be disclosed.}

Open Access This article is distributed under the terms of the Creative Commons Attribution Noncommercial License which permits any noncommercial use, distribution, and reproduction in any medium, provided the original author(s) and source are credited.

\section{References}

1. Matar N, Amoussa K, Verduyckt I, Nollevaux MC, Jamart J, Lawson G, Remacle M (2010) $\mathrm{CO}_{2}$ laser-assisted microsurgery for intracordal cysts: technique and results of 49 patients. Eur Arch Otorhinolaryngol 267:1905-1909

2. Bouwers F, Dikkers FG (2009) A retrospective study concerning the psychosocial impact of voice disorders: voice handicap index change in patients with benign voice disorders after treatment (measured with the Dutch version of the VHI). J Voice 23:218-224

3. Benninger MS (2009) Laser surgery for nodules and other benign laryngeal lesions. Curr Opin Otolaryngol Head Neck Surg 17: 440-444
F. G. Dikkers $(\square)$

Department of Otorhinolaryngology, University Medical Center Groningen, University of Groningen, P.O. Box 30001 , 9700 RB Groningen, The Netherlands e-mail: f.g.dikkers@kno.umcg.nl 